\title{
IL-33 blockade suppresses tumor growth of human lung cancer through direct and indirect pathways in a preclinical model
}

\author{
Kailing Wang ${ }^{1, *}$, Shan Shan ${ }^{1,3, *}$, Zongjun Yang ${ }^{2}$, Xia Gu ${ }^{1}$, Yuanyuan Wang ${ }^{1}$, Chunhong \\ Wang ${ }^{1}$ and Tao $\operatorname{Ren}^{1,3}$ \\ ${ }^{1}$ Department of Respiratory Medicine, East Hospital, Tongji University School of Medicine, Shanghai 200120, China \\ ${ }^{2}$ Department of Clinical Laboratory, Qingdao Women \& Children Hospital, Qingdao 266034, China \\ ${ }^{3}$ Department of Respiratory Medicine, Shanghai Jiao Tong University Affiliated Sixth People's Hospital, Shanghai 200233, \\ China \\ * These authors have contributed equally to this work \\ Correspondence to: Tao Ren, email: rentaosh@126.com \\ Chunhong Wang, email: wangch910@126.com \\ Keywords: lung cancer, IL-33, tumor-associated macrophage, regulatory T cell \\ Abbreviations: NSCLC: non-small-cell lung cancer; TAM: tumor-associated macrophage; Treg: regulatory T cell \\ Received: February 18, $2017 \quad$ Accepted: June 18, $2017 \quad$ Published: August 02, 2017 \\ Copyright: Wang et al. This is an open-access article distributed under the terms of the Creative Commons Attribution License 3.0 \\ (CC BY 3.0), which permits unrestricted use, distribution, and reproduction in any medium, provided the original author and source \\ are credited.
}

\section{ABSTRACT}

Non-small-cell lung cancer (NSCLC) is the most common type in lung cancer, a leading cause of cancer-related death worldwide. Our previous study unraveled a procancer function of IL-33 in fueling outgrowth and metastasis of human NSCLC cells. Herein, we determined that interfere with IL-33 activity was an effective strategy for limiting NSCLC tumor growth using a preclinical model with human NSCLC xenografts. IL-33 blockade efficiently inhibited tumor growth of NSCLC xenografts in immunedeficient mice. Mechanistically, IL-33 blockade suppressed outgrowth capacity of human NSCLC cells. Meanwhile, IL-33 blockade abrogated polarization of M2 tumorassociated macrophages (TAMs) and reduced accumulation of regulatory $\mathrm{T}$ cells (Tregs) in tumor microenvironments, shaping functional immune surveillance. In NSCLC patients, IL-33 expressions were positively correlated with Ki-67 proliferation index and expressions of M2 TAM- and Teg-related genes. These findings identify IL33 as a dual-functional factor in NSCLC pathogenesis and suggest IL-33 blockade as a promising therapeutic for NSCLC patients.

\section{INTRODUCTION}

Lung cancer is the leading cause of cancer-related death worldwide [1]. Among patients with lung cancer, non-small cell lung cancer (NSCLC) accounts for about $85 \%$ of all clinical cases $[1,2]$. Besides the traditional therapeutics including surgery and chemotherapy, targeted therapy is currently considered as an optimal treatment for NSCLC patients $[3,4]$. The application of targeted drugs is based on accurate NSCLC genetic mutations, which are crucial for tumor initiation and progression [3-5]. However, the majority of NSCLC patients are wild type and not suitable be treated by targeted drugs [6]. Resistance to targeted drugs seems common and inevitable, dampening the effectiveness of targeted therapy [6-8]. Currently, the prognoses of NSCLC patients are far less satisfied [9]. New therapeutic explorations are urgently needed for optimizing treatment of NSCLC patients.

Interleukin-33 (IL-33) is one new member of IL-1 superfamily and functions either as a nuclear transcriptional factor or as a released cytokine $[10,11]$. IL-33 maintains barrier functions as a nuclear protein in physiological conditions and participates in pathogenic diseases as an alarming cytokine by binding to its receptor ST2 [10-12]. Recent studies identify IL-33 as an important pro-cancer factor. For instance, IL-33 is able to promote the growth and metastasis of colorectal cancer, breast cancer, gastric cancer and ovarian cancer [13-19]. 
In consistent, our previous study reported a crucial role of IL-33 in driving NSCLC outgrowth and metastasis through regulating membrane GLUT1 localization and aerobic glycolysis, suggesting IL-33 as a promising target for treatment of NSCLC patients [20].

Current studies investigating therapeutic efficiency of anti-cancer reagents are heavily relied on murine models with established human tumor cell lines, frequently resulting in difficulties from translating the findings into clinical application because tumor cell lines do not maintain the characteristics and heterogeneity of cancer cells from clinical patients $[21,22]$. Herein, we evaluate the application of IL-33 based therapeutic in treating NSCLC patients using a pre-clinical mouse model. By engraftment of tumor tissues from NSCLC patients into immune-deficient mice and subsequent treatment of tumor-bearing mice with IL-33 blockers, we demonstrate that blockade of IL-33 is an efficient strategy to limit NSCLC growth. The mechanism involves direct effect by dampening NSCLC proliferative survival and indirect effect by inhibiting polarization of M2 macrophages and reducing accumulation of regulatory $\mathrm{T}$ cells in tumor microenvironments. These findings obtained from human NSCLC tumor xenograft model reflect a realistic condition in vivo, unraveling IL-33 blockade as a novel option for optimizing treatment of NSCLC patients.

\section{RESULTS}

\section{Establishment of patient-derived NSCLC tumor xenograft model}

Fresh NSCLC tumor fragments from 22 patients were implanted subcutaneously into SCID mice and monitored for tumor growth. The tumor xenografts removed and passaged to nude mice were established from 6 patient-derived samples, showing stable tumor growth in nude mice in the third generation (Figure 1). These established NSCLC xenograft models were used to evaluate the efficacy of IL-33 based therapeutics.

\section{IL-33 blockade limits tumor growth of NSCLC tumor xenograft}

To determine the effect of IL-33 based therapeutics in treatment of NSCLC, nude mice bearing the fourth generation of human NSCLC tumors were treated with recombinant IL-33 protein plus IL-33 neutralizing antibody or ST2 neutralizing antibody. While tumor growth of untreated NSCLC xenografts was stable in vivo, it was significantly enhanced by IL-33 treatment (Figure 2A). The function of IL-33 in promoting NSCLC xenograft growth could be abrogated by IL-33 neutralizing antibody and ST2 neutralizing antibody (Figure 2B, 2C). Of note, IL-33 blockade efficiently alleviated tumor growth of untreated NSCLC xenografts (Figure 2D, $2 \mathrm{E}$ ). These data suggest IL-33 blockade as an effective therapeutic strategy for human NSCLC.

\section{IL-33 blockade inhibits proliferative survival of NSCLC cells}

To understand the mechanisms underlying limited NSCLC growth by IL-33 blockade, freshly isolated NSCLC cells from surgical tissues were incubated with recombinant human IL-33 protein with or without IL-33 neutralizing antibody and analyzed for their proliferative survival by MTT assay. IL-33 promoted outgrowth capacity of human NSCLC cells, which could be blocked by IL-33 neutralization (Figure 3A, 3B). Further, IL33 neutralization itself exerted an inhibitory function in NSCLC proliferative survival (Figure 3C), suggesting that

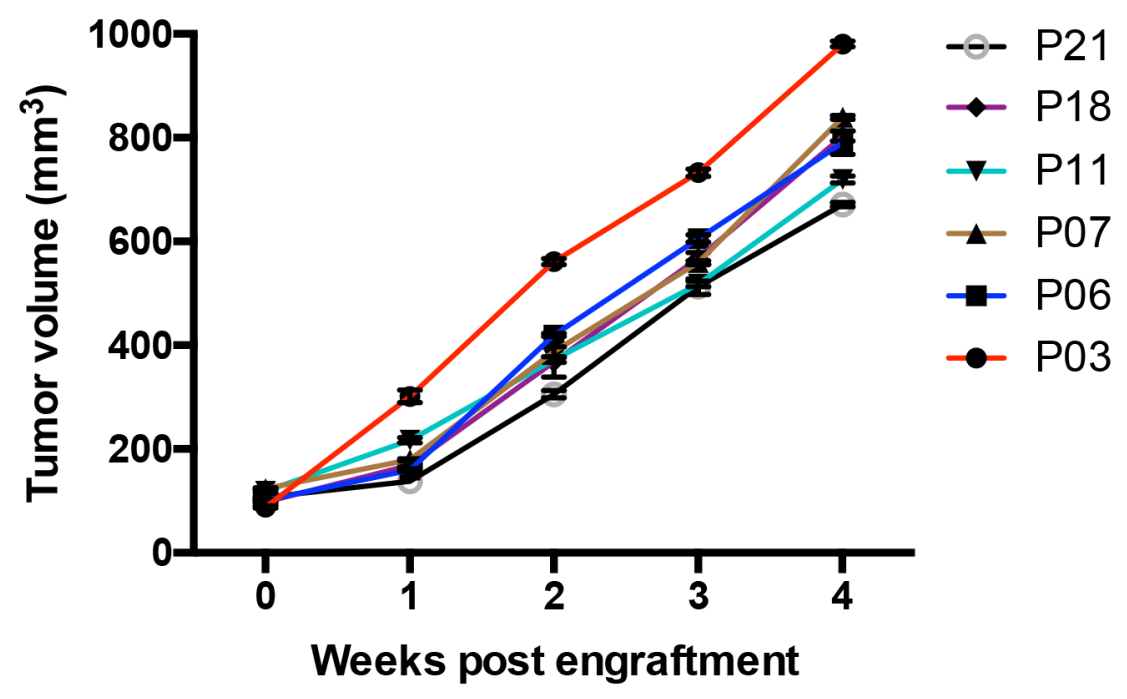

Figure 1: Tumor growth of human NSCLC xenografts. Tumor volumes of NSCLC xenografts in the third generation were detected at the indicated time post engraftment. Each tumor was measured by two researchers. Shown are mean \pm SEM from 6 patients samples. 
IL-33 promotes NSCLC outgrowth through an autocrine manner. These data were in line with our previous study that showed a robust expression of IL-33 and ST2 in tumor cells of NSCLC patients [20]. In contrast, we did not observe a significant effect of IL-33 blockade on NSCLC apoptosis (Figure 3D, 3E). Collectively, IL-33 blockade suppresses NSCLC progression, at least partly, through a direct inhibition on NSCLC outgrowth.

A

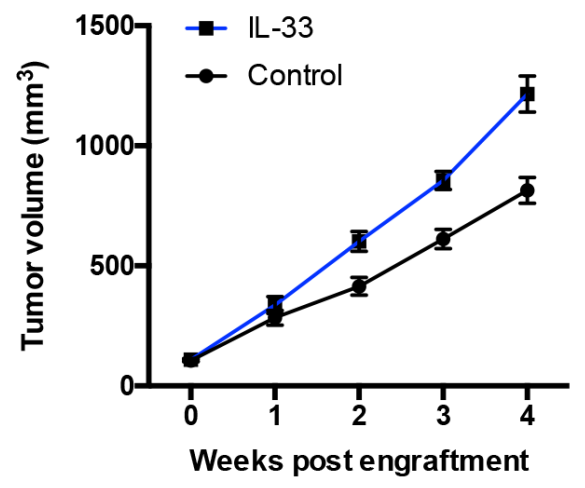

B

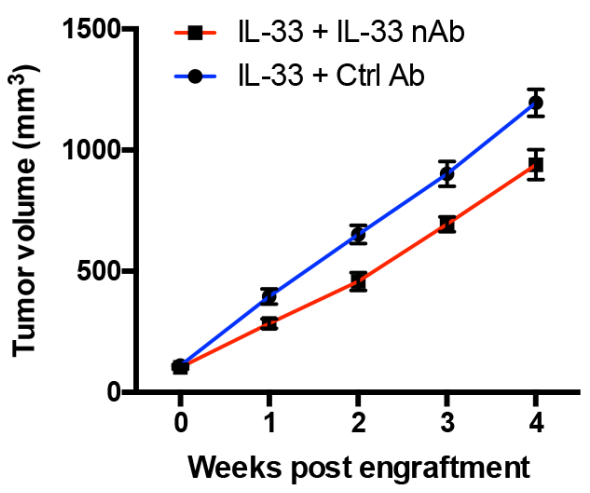

D

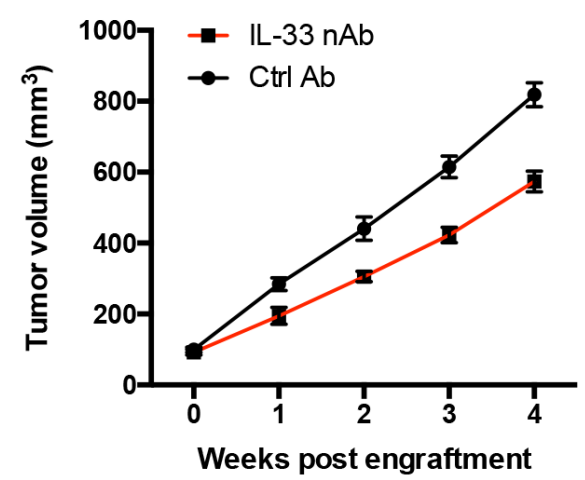

\section{IL-33 blockade abrogates polarization of M2 tumor-associated macrophages in tumor microenvironment}

In NSCLC tumor tissues, IL-33 is robustly expressed in cancer cells while ST2 is expressed on both cancer cells and infiltrated lymphocytes [20], indicating IL-33 as a dual-function factor that could affect both NSCLC cells
C

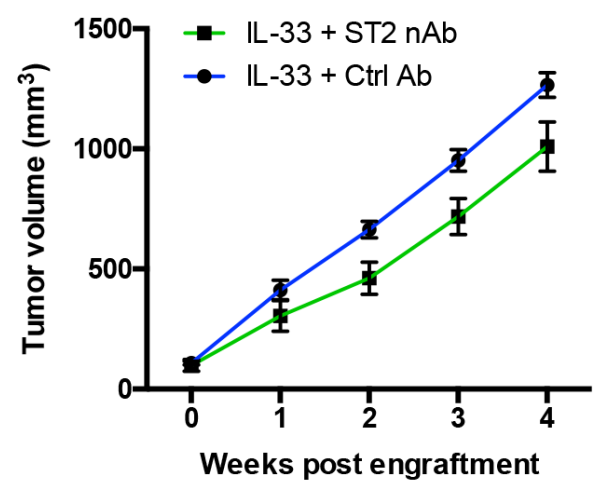

E

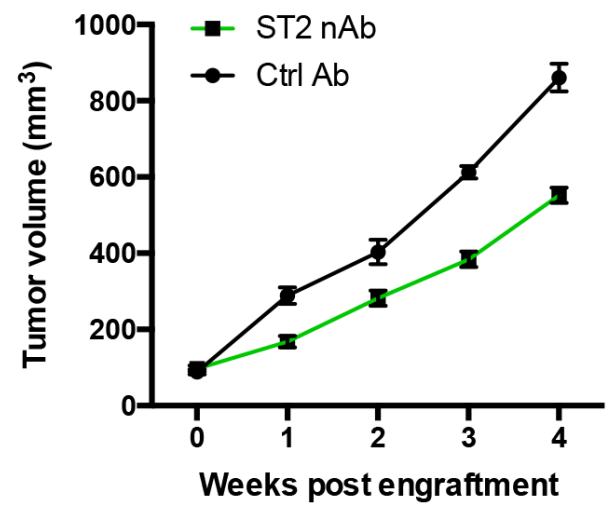

Figure 2: IL-33 blockade restricts tumor growth of NSCLC xenografts. (A) NSCLC xenografts were treated with recombinant human IL-33 protein or the control and detected for tumor growth at the indicated time. (B, C) NSCLC xenografts were treated with recombinant human IL-33 protein plus IL-33 neutralizing antibody (B) or ST2 neutralizing antibody (C). (D, E) NSCLC xenografts were treated with IL-33 neutralizing antibody (D) or ST2 neutralizing antibody (E) and analyzed for tumor growth. Shown are mean \pm SEM from 6 independent experiments. 
and immune surveillance in tumor microenvironments. Thus, freshly isolated tumor-infiltrating lymphocytes (TILs) and NSCLC cells were co-cultured to mimic the tumor microenvironment and analyzed for polarization of M2-polarized tumor-associated macrophages (TAMs), which is critical for tumor progression [23, 24]. Incubation with NSCLC cells enhanced the polarization

A

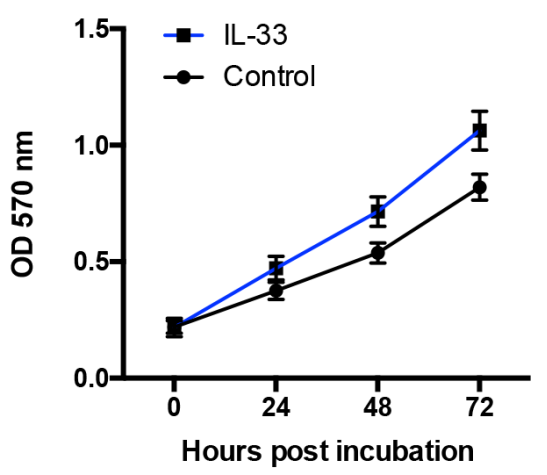

C

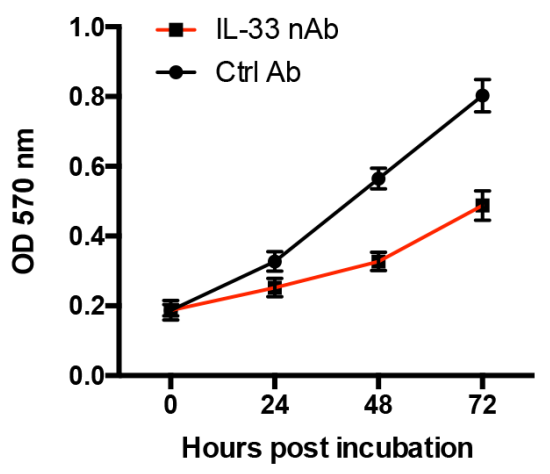

D

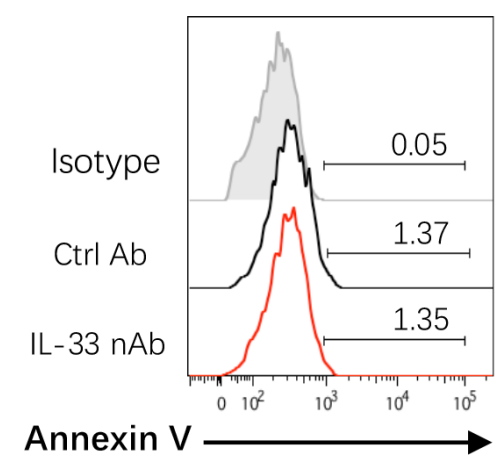

of CD163-positive M2 TAMs in TILs, accompanied by an increased production of IL-10 (Figure 4A-4B and Supplemenatry Figure 1). The effect of NSCLC cells on polarization of M2 TAMs could be abrogated by IL-33 neutralization (Figure 4C and Supplemenatry Figure 1). To further confirm these findings in vivo, NSCLC tumor fragments were implanted into immune-deficient NSG

\section{B}

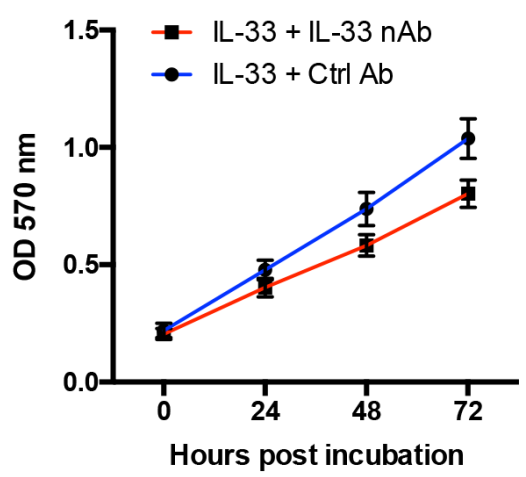

E

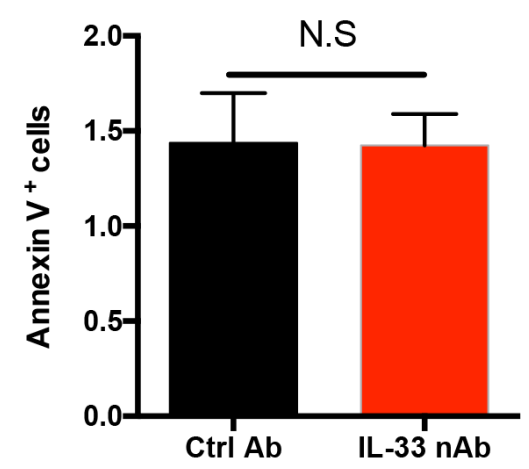

Figure 3: IL-33 confers NSCLC proliferative expansion. (A) Freshly isolated NSCLC cells were treated with recombinant human IL-33 protein $(20 \mathrm{ng} / \mathrm{ml})$ or the control and analyzed for their proliferative growth with MTT assay. Shown are mean \pm SEM from 4 independent experiments. (B) Freshly isolated NSCLC cells were treated with recombinant human IL-33 protein (20 ng/ml) plus IL-33 blocking antibody $(10 \mu \mathrm{g} / \mathrm{ml})$ and analyzed for their proliferative growth. Shown are mean \pm SEM from 4 independent experiments. (C) Freshly isolated NSCLC cells were treated with IL-33 blocking antibody $(10 \mu \mathrm{g} / \mathrm{ml})$ or the control and analyzed for their proliferative growth. Shown are mean \pm SEM from 4 independent experiments. (D, E) Freshly isolated NSCLC cells were treated with IL-33 neutralizing antibody $(10 \mu \mathrm{g} / \mathrm{ml})$ or the control antibody for $24 \mathrm{~h}$ and analyzed for their apoptosis with Annexin V staining. Representative histograms and collective data (mean \pm SEM) from 4 independent experiments are shown. 
A

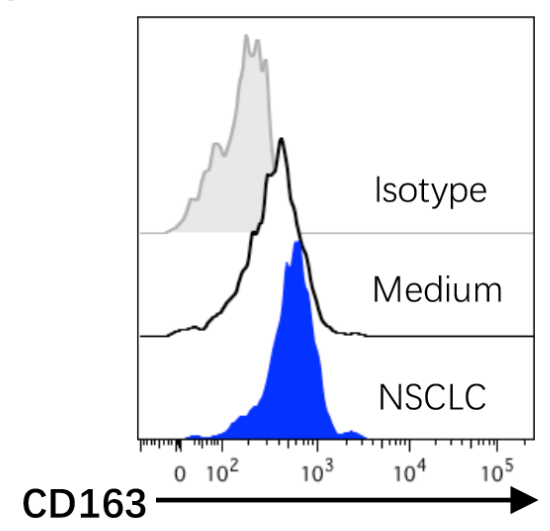

C

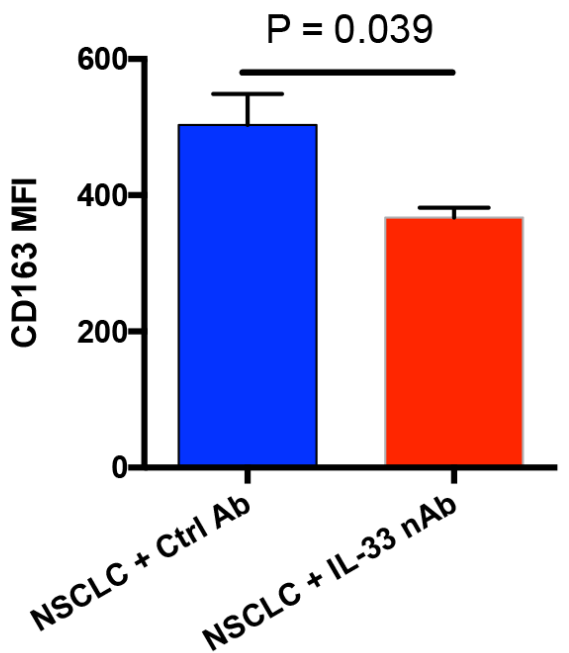

$\mathrm{E}$

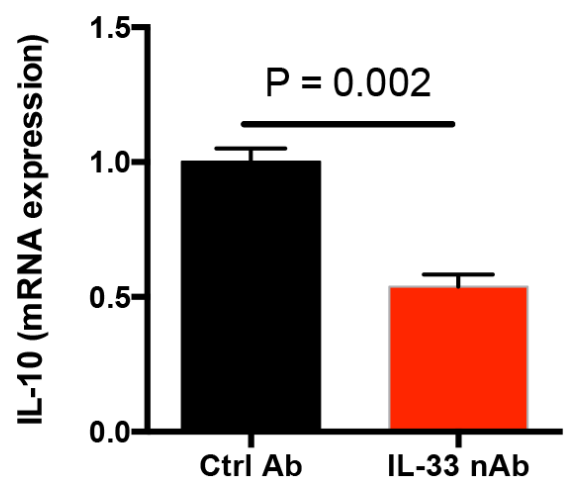

B

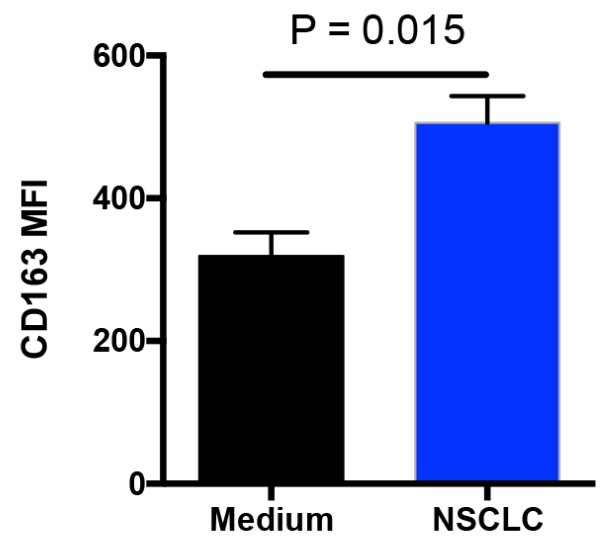

$\mathrm{D}$

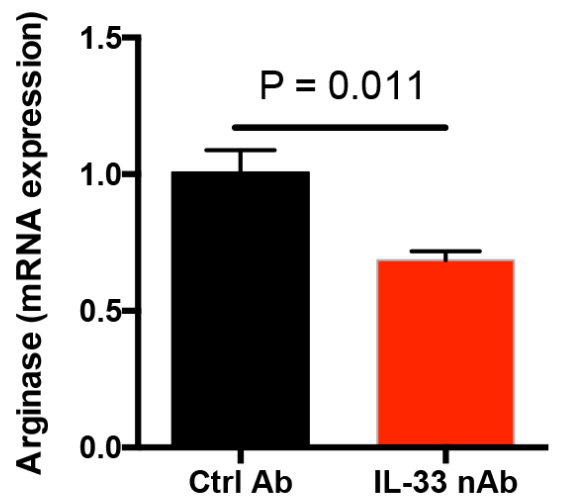

F

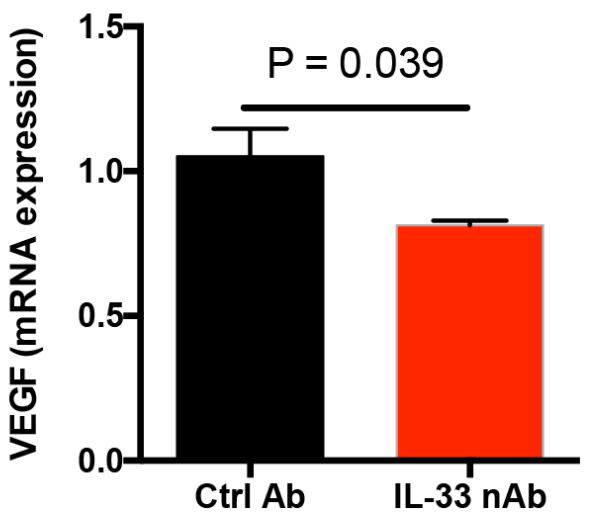

Figure 4: IL-33 blockade abrogates polarization of M2 TAMs in tumor microenvironment. (A, B) Fresh TILs were treated with M-CSF $(10 \mathrm{ng} / \mathrm{ml})$ plus IL-10 $(10 \mathrm{ng} / \mathrm{ml})$ and co-cultured with or without NSCLC cells for 4 days. CD68-positive macrophages were gated from TILs and analyzed for CD163 expressions. Representative histograms and collective data (mean \pm SEM) from 6 independent experiments are shown. (C) Fresh TILs were incubated with M-CSF $(10 \mathrm{ng} / \mathrm{ml}), \mathrm{IL}-10(10 \mathrm{ng} / \mathrm{ml})$ and NSCLC cells in the presence or absence of IL-33 neutralizing antibody $(10 \mu \mathrm{g} / \mathrm{ml})$ for 4 days. CD163 expression on CD68-positive macrophages was detected by flow cytometry. Shown are mean \pm SEM from 6 independent experiments. (D-F) Fresh tumor fragments from NSCLC patients were implanted into NSG mice, treated with IL-33 neutralizing antibody for 7 days and detected for expressions of M2 TAM-related genes including arginase 1 (D), IL-10 (E) and VEGF (F). Shown are mean \pm SEM from 6 independent experiments. 
mice and treated with IL-33 neutralizing antibody. IL33 neutralization significantly inhibited the expressions of M2 TAM-related genes including arginase 1, IL10 and VEGF in tumor tissues (Figure 4D-4F). These findings demonstrate that IL-33 blockade could inhibit the polarization of M2 TAMs, facilitating an anti-cancer tumor microenvironment.

\section{IL-33 blockade reduces accumulation of regulatory $\mathbf{T}$ cells in tumor microenvironment}

Regulatory T cells (Tregs) are the suppressor of anticancer $\mathrm{T}$ effector cells and frequently enriched in tumor microenvironments $[25,26]$. Co-culture with NSCLC cells upregulated the frequency of FoxP3-positive Treg cells in TILs, which could be blocked by IL-33 neutralization (Figure 5A-5D). In vivo confirmation of these data was performed in NSG mice with engraftment of human NSCLC fragments. IL-33 blockade dramatically reduced FoxP3 expression in NSCLC tumor tissues (Figure 5E). In consistent, IL-33 exerted a contributing effect on FoxP3 induction, increasing the frequency of Treg cells in TILs (Figure 5F). The phenomenon was partially dependent on M2 TAMs as it could be abrogated by depletion of macrophages (Figure 5F). It is reasonable because human M2 macrophages could robustly induce Treg differentiation and function [27]. Therefore, diminished Treg cells in tumor tissues by IL-33 blockade could contribute to limited NSCLC growth.

\section{IL-33 expression reflects tumor outgrowth and immune escape features in tumor tissues of NSCLC patients}

To explore the in vivo relevance of above findings, we analyzed the correlation of IL-33 expression with Ki67 proliferation index (PI), and expressions of arginase 1, IL-10 and FoxP3 in tumor tissues of NSCLC patients. We observed significantly higher expressions of IL-33, arginase 1, IL-10 and FoxP3 in NSCLC tumor tissues than adjacent tissues (Figure 6A-6D). Further, IL-33 expression levels were closely associated with expressions of arginase 1 and FoxP3 in NSCLC tissues (Figure 6E, $6 \mathrm{~F})$. IL-33 expression was positively correlated with Ki67 PI of NSCLC patients (Figure 6G). These findings indicate a contributing function of IL-33 in promoting NSCLC proliferative survival, polarization of M2 TAMs and accumulation of Treg cells in NSCLC patients.

\section{DISCUSSION}

In this study, we uncover IL-33 blockade as a novel and effective therapeutic for NSCLC patients using a preclinical model. Blocking IL-33 activity restricts tumor growth of NSCLC xenografts. Mechanistically, IL-33 blockade suppresses outgrowth of NSCLC cells, abrogates polarization of M2 TAMs and reduces accumulation of Treg cells, shaping immune surveillance in tumor microenvironments.

Immunotherapy is a new frontier in clinical treatment of NSCLC patients [28]. The therapeutic is based on the idea that immune surveillance is a crucial mechanism for host protection against carcinogenesis and thus improving the functional competence of immune effector cells would ultimately eliminate tumor cells [29]. Indeed, checkpoint immunotherapies with inhibitors targeting PD-L1/PD-1 have shown exciting results in clinical study [30]. But, cancer incidence increases ever year and cancer develops any way even in the host with a functioning immune system [31]. This opens a complex and dynamic process consisting of immune surveillance and tumor progression, termed as cancer immunoediting $[31,32]$. Herein, we demonstrate that incubation with NSCLC cells facilitated polarization of M2 TAMs and accumulation of Treg cells in tumor microenvironments, suggesting cancer immunoediting as an efficient mechanism for immune escape of lung cancer cells.

IL-33 is expressed in high levels in cancer cells from NSCLC patients, supporting NSCLC outgrowth and metastasis [20]. Here we extend previous findings by providing evidence that IL-33 blockade efficiently limited NSCLC growth through direct and indirect pathways. IL33 neutralization results in reduced proliferative survival of NSCLC cells, insufficient M2-polirzaied TAMs and diminished Treg cells in tumor tissues. In NSCLC patients, IL-33 expression levels were positively correlated with Ki-67 PI and expressions of M2 TAM- and Treg-related genes. In line with our findings, previous studies showed that IL-33 was able to facilitate polarization of M2 macrophages and promote the expansion and function of FoxP3-positive Treg cells [33-37]. Besides, we noticed that increased Treg cells by IL-33 was at least partly dependent on M2 TAMs. Depletion of macrophages significantly abrogated the effect of IL-33 on accumulation of Treg cells. Thus, we proposed an IL-33-M2 TAM-Treg pathway underlying progression of human lung cancer. IL-33 increased the frequency of Treg cells through both M2 TAM-dependent and -independent pathway. Combing these findings suggest IL-33 as a dual-function factor in NSCLC progression. IL-33 functions as an intrinsic molecular mechanism supporting NSCLC outgrowth and a tumor-derived factor involved in cancer immunoediting.

We would like to point out that there are some studies showed an anti-cancer activity of IL-33 using established cancer cell lines and murine models. Qin L et al showed that systemic administration of recombinant IL-33 dramatically inhibited the leukemia growth and prolonged the survival of leukemia-bearing mice by increasing the expansion and IFN- $\gamma$ production of leukemia-reactive CD8 T cells [38]. Tumoral expression of IL-33 was also reported to inhibit tumor growth and modifies the tumor 
microenvironment through CD8 T cells, favoring tumor eradication $[39,40]$. These data raised a possibility that IL-33 might exert anti-cancer activity under certain circumstances. Herein, we determined the pro-cancer activity of IL-33 in tumor progression using fresh isolated cancer cells from lung cancer patients. The distinct observations might due to different types of tumors or reflect a fact that human cancer cell lines could not fully

A

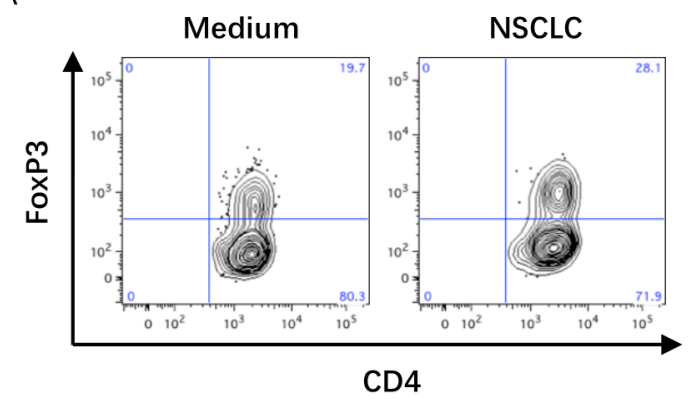

C

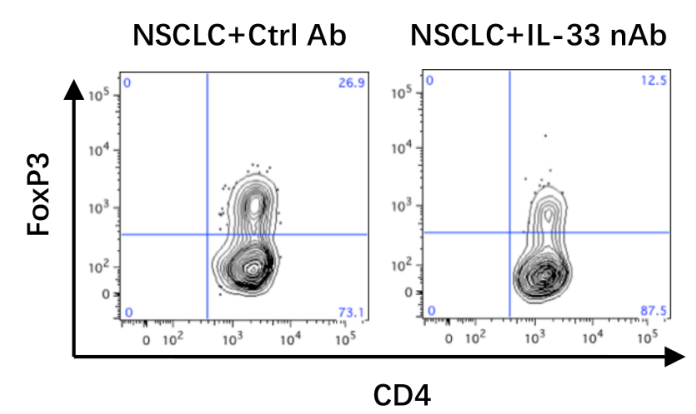

$\mathrm{E}$

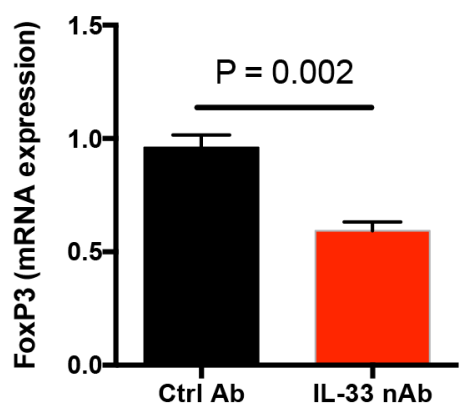

represent the characteristics and heterogeneity of cancer cells from clinical patients.

Our current findings are obtained from a preclinical model with human NSCLC xenografts and thus have a close clinical relevance. IL-33 blockade could be a promising therapeutic strategy for NSCLC patients. However, we need to admit some limitations of this study. First, the sample size is relatively low and experiments

B

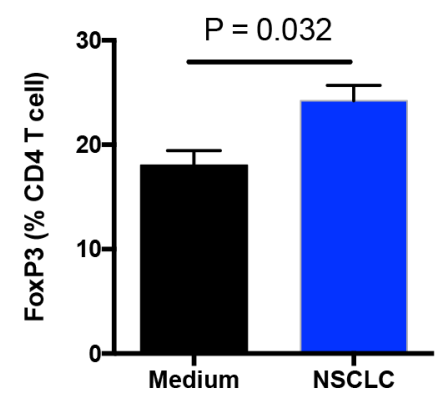

D

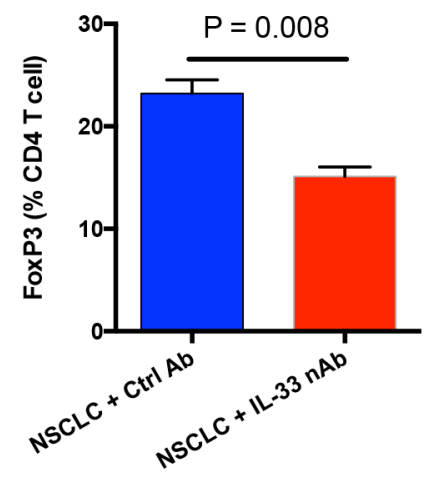

F

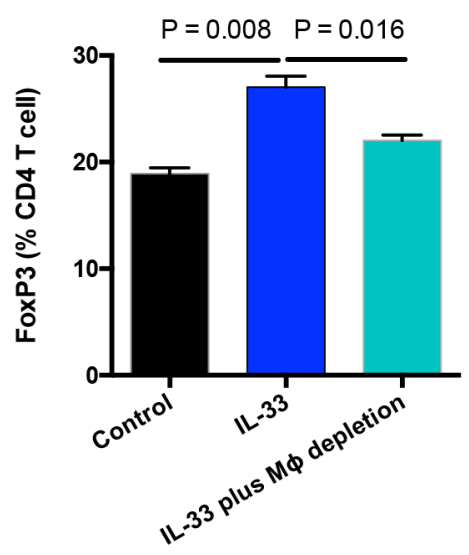

Figure 5: IL-33 blockade reduces Treg accumulation in tumor microenvironment. (A, B) Fresh TILs were stimulated with anti-CD3 antibody $(5 \mu \mathrm{g} / \mathrm{ml})$ plus anti-CD28 antibody $(5 \mu \mathrm{g} / \mathrm{ml})$ and co-cultured with or without NSCLC cells for 4 days. Frequency of FoxP3-expressing CD4 T cells in TILs was analyzed by flow cytometry. Representatives and data (mean \pm SEM) from 5 independent experiments are shown. (C, D) Fresh TILs were incubated with anti-CD3 antibody ( $5 \mu \mathrm{g} / \mathrm{ml})$, anti-CD28 antibody (5 $\mu \mathrm{g} / \mathrm{ml})$ plus NSCLC cells in the presence of IL-33 neutralizing antibody $(10 \mu \mathrm{g} / \mathrm{ml})$ or the control for 4 days. Representatives and data (mean \pm SEM) from 5 independent experiments are shown. (E) Fresh tumor fragments from NSCLC patients were implanted into NSG mice, treated with IL-33 neutralizing antibody for 7 days and detected for FoxP3 expressions. Shown are mean \pm SEM from 6 independent experiments. (F) Fresh TILs with or without depletion of macrophages were stimulated with anti-CD3 plus anti-CD28 antibodies $(5 \mu \mathrm{g} / \mathrm{ml})$ in the presence or absence of IL-33 protein $(20 \mathrm{ng} / \mathrm{ml})$ for 4 days. Shown are mean \pm SEM from 5 independent experiments. 
A

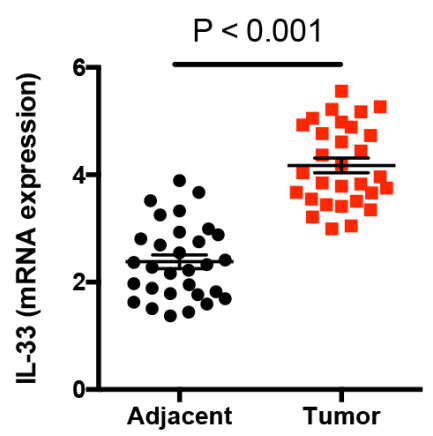

C

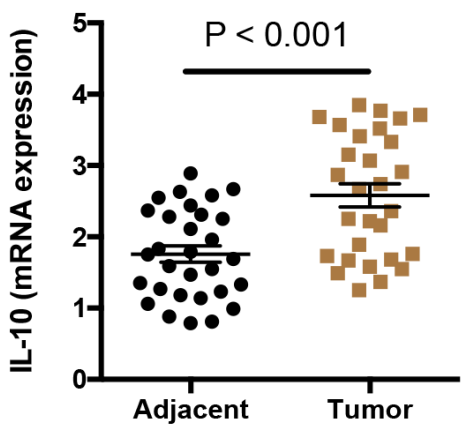

E

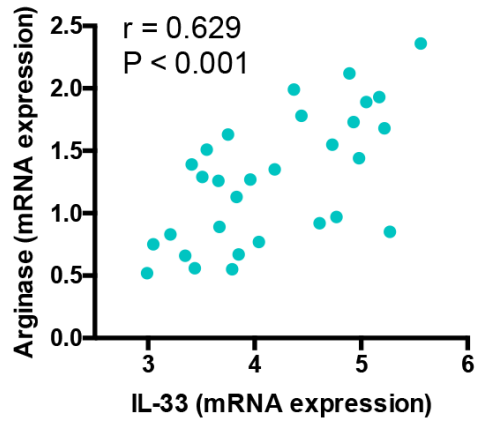

G



B

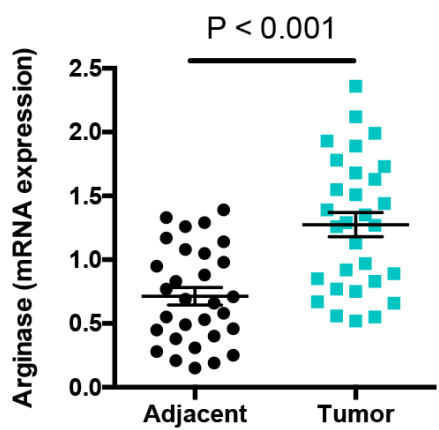

D

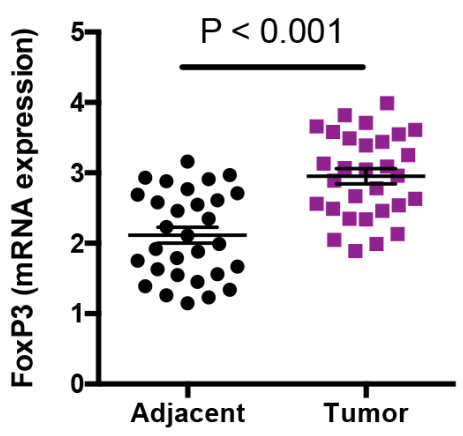

$\mathrm{F}$

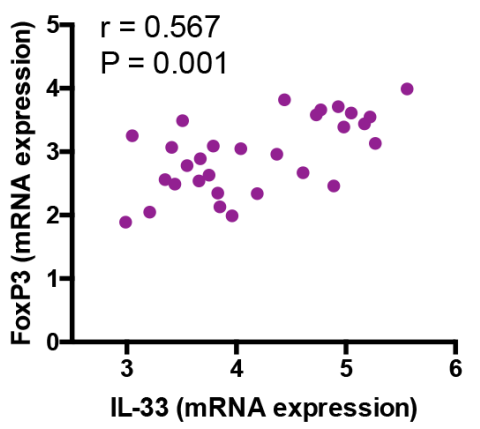

Figure 6: Increased IL-33 expression reflects tumor outgrowth and immune escape features in tumor tissues. (A-D) Expressions of IL-33, arginase 1, IL-10 and FoxP3 were analyzed in tumor tissues and adjacent tissues from NSCLC patients ( $\mathrm{n}=30$ ) by qPCR. Shown are relative expression normalized to the internal control. (E-G) IL-33 expression in tumor tissues was analyzed for correlations with expressions of arginase 1 and FoxP3, and Ki-67 PI in NSCLC patients. Each dot represents the data from an individual patient. 
on a large size of NSCLC samples would be valuable to substantiate these findings. Second, although the preclinical model with NSCLC xenografts in immunedeficient mice closely reflects an in vivo relevance and provides a useful platform to explore anti-cancer reagents, this model could not represent the complexity of NSCLC progression in clinical patients. In addition, precise molecular mechanisms connecting IL-33 and tumor outgrowth, as well as immune surveillance, in NSCLC patients are unclear. Therefore, there is still a long distance from current findings to clinical applications.

In essence, NSCLC-derived IL-33 supports tumor growth in an autocrine manner and educates immune surveillance in tumor microenvironments, favoring immune escape of tumor cells. IL-33 blockade restricts NSCLC outgrowth, abrogates polarization of M2 TAMs and reduces accumulations of Treg cells in tumor tissues, representing an effective and promising strategy for NSCLC treatment. These findings shed new insight into NSCLC pathogenesis and provide clues for developing novel therapeutics against NSCLC in clinical practice.

\section{MATERIALS AND METHODS}

\section{Patients}

Thirty-five patients with biopsy-proven diagnosis of NSCLC were enrolled in this study. Written informed consent was obtained from each individual patient before collecting surgical tissues and clinical parameters. Ki-67 proliferation index was evaluated by two pathologists in our institution. The clinical parameters of NSCLC patients were summarized in Supplementary Table 1. Experiments were performed in accordance with the 1964 Helsinki declaration including its later amendments and approved by Tongji Institutional Ethics Committee.

\section{Mice}

Immune-deficient SCID mice, nude mice and NSG mice between 6 to 8 weeks of age were from Shanghai Laboratory Animal Center, CAS and Biocytogen LLC. Mice were housed under specific pathogen-free conditions and experiments were approved by Tongji Institutional Ethics Committee.

\section{Cells and reagents}

NSCLC cells were isolated from surgical tissues with Clonogenic Tumor Cell Isolation Kit (Cell Biolabs) [20]. Digestion of NSCLC tissues was performed as previously described [41], and tumor infiltrating lymphocytes (TILs) were isolated with CD45 MicroBeads, human (Miltenyi Biotec). Depletion of macrophages from TILs was performed by using CD14 Microbeads, Human (Miltenyi Biotec). Cells were cultured in complete RPMI 1640 medium containing 10\% heat-inactivated FBS
(Gibco) supplemented with 2mM glutamine, 100IU/ml penicillin and $100 \mathrm{mg} / \mathrm{ml}$ streptomycin sulfate at $37^{\circ} \mathrm{C}$ under 5\% CO2. Kits were used according to the manual's instructions.

Recombinant human IL-33 protein, human M-CSF protein, human IL-10 protein, human IL-33 neutralizing antibody and human ST2 neutralizing antibody were from R\&D Systems. Anti-human CD3 antibody and anti-human CD28 antibody were from eBioscience. All reagents were used according to manufacturer's instructions.

\section{NSCLC xenografts and treatment}

Establishment of NSCLC xenografts in immunedeficient mice were performed as previously described [21, $22,42]$. Fresh tumor samples from NSCLC patients were cut into pieces of $4-5 \mathrm{~mm}$ and implanted subcutaneously into immune-deficient SCID mice. Tumor growth was measured with digital calipers by two researchers every week and tumors were removed into immune-deficient nude mice when the tumor size reached $500-600 \mathrm{~mm}^{3}$. The mouse-to-mouse passage was performed to establish stable growth of NSCLC xenografts.

NSCLC xenografts in the fourth generation were used to evaluate therapeutic efficiency of IL-33 blockade. Mice bearing NSCLC xenografts were injected intraperitoneally with $1 \mu \mathrm{g}$ of human IL-33 protein, $100 \mu \mathrm{g}$ of IL-33 neutralizing antibody or $100 \mu \mathrm{g}$ of ST2 neutralizing antibody at three times a week for 4 weeks $[43,44]$. Tumor size were measured with digital calipers by two researchers every week.

\section{MTT assay}

NSCLC outgrowth capacity was detected by MTT assay [20]. NSCLC cells $\left(6 \times 10^{3}\right.$ cells/well $)$ were cultured with recombinant human IL-33 protein in the presence or absence of IL-33 neutralizing antibody and measured with MTT cell proliferation kit (Cayman Chemical) according to the manual's instructions.

\section{Transwell co-culture assay}

Co-culture of TILs with NSCLC cells was performed with the trans-well assay [45]. Freshly isolated TILs $\left(5 \times 10^{5}\right.$ cells $)$ were placed in the lower compartment of Corning ${ }^{\circledR}$ Transwell ${ }^{\circledR}$ polyester membrane cell culture inserts. Same number of NSCLC cells were seeded in the upper compartment and co-cultured with TILs for 4 days. TILs were then collected for analyzing macrophages and Treg cells with flow cytometry.

\section{Flow cytometry}

To analyze apoptosis of NSCLC cells, $2 \times 10^{5}$ cells were stained for Annexin V with Annexin V-FITC Apoptosis Detection Kit (eBioscience) according to the 
manual's instructions. For detecting the frequencies of M2TAMs and regulatory T cells, $2 \times 10^{5}$ cells were treated with cell fix/perm buffer (BD Bioscience) and stained with FITC anti-human CD68 antibody, APC anti-human CD163 antibody, PE-Cy7 anti-human CD4 antibody, PE anti-human FoxP3 antibody or isotype controls (all from eBioscience) for $45 \mathrm{~min}$ at room temperature in the dark. Samples were analyzed on a LSR II flow cytometer (BD Biosciences) and data were analyzed with FlowJo software (Tree Star).

\section{Real-time PCR}

Detection of mRNA expressions of human IL33, arginase 1, VEGF and Foxp3 was performed using quantitative real-time PCR [20]. Total RNA was extracted using Ambion ${ }^{\circledR}$ Isolation Kit (Thermo Fisher). cDNA was synthesized with First-Strand cDNA Synthesis Kit (Thermo Fisher). qPCR was performed using SYBR Green Master Mixes (Thermo Fisher). Human IL-33, arginase 1, VEGFA, Foxp3 and GAPDH primers were obtained from Sino Biological Inc. GAPDH was used as an internal control.

\section{Statistical analyses}

Data were presented as mean \pm SEM. MannWhitney test and Spearman correlation were used for statistical analyses using the program PRISM 6.0 (GraphPad Software Inc). A value of $\mathrm{P}<0.05$ was considered statistically significant.

\section{Author contributions}

K.W and S.S performed experiments. Z.Y and X.G assisted experimental work and collected data. Y.W analyzed data. C.W and T.R designed the study and wrote the manuscript. All authors have reviewed and agreed to this information.

\section{CONFLICTS OF INTEREST}

The authors declare no conflicts of interest.

\section{FUNDING}

This work was funded by grants from National Natural Science Foundation of China (81402555, 81673014, 81372347), Outstanding Academic Leaders Plan of Shanghai Municipal Science and Technology Committee (16XD1403100) and National Key R\&D Plan (2017YFA0104600). The funding provided financial support without any involvement in study design, data analysis and manuscript preparation.

\section{REFERENCES}

1. Torre LA, Bray F, Siegel RL, Ferlay J, Lortet-Tieulent J, Jemal A. Global cancer statistics, 2012. CA Cancer J Clin. 2015; 65: 87-108.

2. Langevin SM, Kratzke RA, Kelsey KT. Epigenetics of lung cancer. Transl Res. 2015; 165: 74-90.

3. Dholaria B, Hammond W, Shreders A, Lou Y. Emerging therapeutic agents for lung cancer. J Hematol Oncol. 2016; 9: 138 .

4. Gandara DR, Riess JW, Kelly K, Li T, Mack PC, Lara PN Jr. Evolution and increasing complexity of the therapeutic landscape in advanced non-small-cell lung cancer. Clin Lung Cancer. 2017; 18: 1-4.

5. Castellanos E, Feld E, Horn L. Driven by Mutations: The Predictive Value of Mutation Subtype in EGFR-Mutated Non-Small Cell Lung Cancer. J Thorac Oncol. 2017; 12: 612-623.

6. Cortinovis D, Gregorc V, Migliorino MR, Abate MI, Manzo A, Malapelle U, Morabito A; Italian Lung Cancer Working Group. New perspectives in the second-line treatment of non squamous NSCLC patients: results from a large Italian Lung Cancer Working Group. Crit Rev Oncol Hematol. 2017; 109: 35-41.

7. Zulkifli AA, Tan FH, Putoczki TL, Stylli SS, Luwor RB. STAT3 signaling mediates tumour resistance to EGFR targeted therapeutics. Mol Cell Endocrinol. 2017; 451: $15-23$.

8. Martinelli E, Morgillo F, Troiani T, Ciardiello F. Cancer resistance to therapies against the EGFR-RAS-RAF pathway: the role of MEK. Cancer Treat Rev. 2016; 53: 61-69.

9. Simmons CP, Koinis F, Fallon MT, Fearon KC, Bowden J, Solheim TS, Gronberg BH, McMillan DC, Gioulbasanis I, Laird BJ. Prognosis in advanced lung cancer-a prospective study examining key clinicopathological factors. Lung Cancer. 2015; 88: 304-309.

10. Hardman C, Ogg G. Interleukin-33, friend and foe in type-2 immune responses. Curr Opin Immunol. 2016; 42: 16-24.

11. Liew FY, Girard JP, Turnquist HR. Interleukin-33 in health and disease. Nat Rev Immunol. 2016; 16: 676-689.

12. Martin NT, Martin MU. Interleukin 33 is a guardian of barriers and a local alarmin. Nat Immunol. 2016; 17: 122-131.

13. Schwartz C, O'Grady K, Lavelle EC, Fallon PG. Interleukin 33: an innate alarm for adaptive responses beyond Th2 immunity-emerging roles in obesity, intestinal inflammation, and cancer. Eur J Immunol. 2016; 46: 1091-1100.

14. Zhang Y, Davis C, Shah S, Hughes D, Ryan JC, Altomare $\mathrm{D}$, Peña MM. IL-33 promotes growth and liver metastasis of colorectal cancer in mice by remodeling the tumor microenvironment and inducing angiogenesis. Mol Carcinog. 2017; 56: 272-287. 
15. Liu X, Zhu L, Lu X, Bian H, Wu X, Yang W, Qin Q. IL-33/ ST2 pathway contributes to metastasis of human colorectal cancer. Biochem Biophys Res Commun. 2014; 453: 486-492.

16. Kim JY, Lim SC, Kim G, Yun HJ, Ahn SG, Choi HS. Interleukin-33/ST2 axis promotes epithelial cell transformation and breast tumorigenesis via upregulation of COT activity. Oncogene. 2015; 34: 4928-4938.

17. Jovanovic IP, Pejnovic NN, Radosavljevic GD, Pantic JM, Milovanovic MZ, Arsenijevic NN, Lukic ML. Interleukin-33/ST2 axis promotes breast cancer growth and metastases by facilitating intratumoral accumulation of immunosuppressive and innate lymphoid cells. Int J Cancer. 2014; 134: 1669-1682.

18. Deng K, Wang H, Shan T, Chen Y, Zhou H, Zhao Q, Xia J. Tristetraprolin inhibits gastric cancer progression through suppression of IL-33. Sci Rep. 2016; 6: 24505.

19. Tong X, Barbour M, Hou K, Gao C, Cao S, Zheng J, Zhao $\mathrm{Y}, \mathrm{Mu} \mathrm{R}$, Jiang HR. Interleukin-33 predicts poor prognosis and promotes ovarian cancer cell growth and metastasis through regulating ERK and JNK signaling pathways. Mol Oncol. 2016; 10: 113-125.

20. Wang C, Chen Z, Bu X, Han Y, Shan S, Ren T, Song W. IL-33 signaling fuels outgrowth and metastasis of human lung cancer. Biochem Biophys Res Commun. 2016; 479: 461-468.

21. Zhang XC, Zhang J, Li M, Huang XS, Yang XN, Zhong WZ, Xie L, Zhang L, Zhou M, Gavine P, Su X, Zheng L, Zhu G, et al. Establishment of patient-derived nonsmall cell lung cancer xenograft models with genetic aberrations within EGFR, KRAS and FGFR1: useful tools for preclinical studies of targeted therapies. J Transl Med. 2013; 11: 168.

22. Tentler JJ, Tan AC, Weekes CD, Jimeno A, Leong S, Pitts TM, Arcaroli JJ, Messersmith WA, Eckhardt SG. Patientderived tumour xenografts as models for oncology drug development. Nat Rev Clin Oncol. 2012; 9: 338-350.

23. Marchesi F, Cirillo M, Bianchi A, Gately M, Olimpieri OM, Cerchiara E, Renzi D, Micera A, Balzamino BO, Bonini S, Onetti Muda A, Avvisati G. High density of CD68+/ CD163+ tumour-associated macrophages (M2-TAM) at diagnosis is significantly correlated to unfavorable prognostic factors and to poor clinical outcomes in patients with diffuse large B-cell lymphoma. Hematol Oncol. 2015; 33: 110-112.

24. Yuan A, Hsiao YJ, Chen HY, Chen HW, Ho CC, Chen YY, Liu YC, Hong TH, Yu SL, Chen JJ, Yang PC. Opposite effects of M1 and M2 macrophage subtypes on lung cancer progression. Sci Rep. 2015; 5: 14273.

25. Tanaka A, Sakaguchi S. Regulatory $\mathrm{T}$ cells in cancer immunotherapy. Cell Res. 2017; 27: 109-118.

26. Wang K, Vella AT. Regulatory T cells and cancer: a twosided story. Immunol Invest. 2016; 45: 797-812.
27. Schmidt A, Zhang XM, Joshi RN, Iqbal S, Wahlund C, Gabrielsson S, Harris RA, Tegnér J. Human macrophages induce $\mathrm{CD} 4(+)$ Foxp3 $(+)$ regulatory T cells via binding and re-release of TGF- $\beta$. Immunol Cell Biol. 2016; 94: 747-762.

28. Bansal P, Osman D, Gan GN, Simon GR, Boumber Y. Recent advances in immunotherapy in metastatic NSCLC. Front Oncol. 2016; 6: 239.

29. D'Errico G, Machado HL, Sainz B Jr. A current perspective on cancer immune therapy: step-by-step approach to constructing the magic bullet. Clin Transl Med. 2017; 6: 3 .

30. Tanvetyanon T, Gray JE, Antonia SJ. PD-1 checkpoint blockade alone or combined PD-1 and CTLA-4 blockade as immunotherapy for lung cancer? Expert Opin Biol Ther. 2017; 17: 305-312.

31. Ribatti D. The concept of immune surveillance against tumors. The first theories. Oncotarget. 2017; 8: 7175-7180. https://doi.org/10.18632/oncotarget.12739.

32. Kim R, Emi M, Tanabe K. Cancer immunoediting from immune surveillance to immune escape. Immunology. 2007; 121: 1-14.

33. Wang C, Dong C, Xiong S. IL-33 enhances macrophage M2 polarization and protects mice from CVB3-induced viral myocarditis. J Mol Cell Cardiol. 2016; 103: 22-30.

34. Peng H, Zhang Q, Li X, Liu Z, Shen J, Sun R, Wei J, Zhao J, Wu X, Feng F, Zhong S, Sun X, Wu Z. IL-33 Contributes to Schistosoma japonicum-induced Hepatic Pathology through Induction of M2 Macrophages. Sci Rep. 2016; 6: 29844.

35. Besnard AG, Guabiraba R, Niedbala W, Palomo J, Reverchon F, Shaw TN, Couper KN, Ryffel B, Liew FY. IL-33-mediated protection against experimental cerebral malaria is linked to induction of type 2 innate lymphoid cells, M2 macrophages and regulatory T cells. PLoS Pathog. 2015; 11: e1004607.

36. Matta BM, Turnquist HR. Expansion of regulatory T cells in vitro and in vivo by IL-33. Methods Mol Biol. 2016; 1371: 29-41.

37. Schiering C, Krausgruber T, Chomka A, Fröhlich A, Adelmann K, Wohlfert EA, Pott J, Griseri T, Bollrath J, Hegazy AN, Harrison OJ, Owens BM, Löhning M, et al. The alarmin IL-33 promotes regulatory T-cell function in the intestine. Nature. 2014; 513: 564-568.

38. Qin L, Dominguez D, Chen S, Fan J, Long A, Zhang M, Fang D, Zhang Y, Kuzel TM, Zhang B. Exogenous IL-33 overcomes $\mathrm{T}$ cell tolerance in murine acute myeloid leukemia. Oncotarget. 2016; 7: 61069-61080. https://doi. org/10.18632/oncotarget.11179.

39. Gao K, Li X, Zhang L, Bai L, Dong W, Gao K, Shi G, Xia X, Wu L, Zhang L. Transgenic expression of IL-33 activates CD8(+) T cells and NK cells and inhibits tumor growth and metastasis in mice. Cancer Lett. 2013; 335: 463-471.

40. Gao X, Wang X, Yang Q, Zhao X, Wen W, Li G, Lu J, Qin W, Qi Y, Xie F, Jiang J, Wu C, Zhang X, et al. Tumoral expression of IL-33 inhibits tumor growth and modifies the 
tumor microenvironment through CD8+ T and NK cells. J Immunol. 2015; 194: 438-445.

41. Baldan V, Griffiths R, Hawkins RE, Gilham DE. Efficient and reproducible generation of tumour-infiltrating lymphocytes for renal cell carcinoma. Br J Cancer. 2015; 112: 1510-1518.

42. Fichtner I, Rolff J, Soong R, Hoffmann J, Hammer S, Sommer A, Becker M, Merk J. Establishment of patientderived non-small cell lung cancer xenografts as models for the identification of predictive biomarkers. Clin Cancer Res. 2008; 14: 6456-6468.

43. Sattler S, Ling GS, Xu D, Hussaarts L, Romaine A, Zhao H, Fossati-Jimack L, Malik T, Cook HT, Botto M, Lau YL, Smits HH, Liew FY, Huang FP. IL-10-producing regulatory
B cells induced by IL-33 (Breg(IL-33)) effectively attenuate mucosal inflammatory responses in the gut. J Autoimmun. 2014; 50: 107-122.

44. Zhang J, Ramadan AM, Griesenauer B, Li W, Turner MJ, Liu C, Kapur R, Hanenberg H, Blazar BR, Tawara I, Paczesny S. ST2 blockade reduces SST2-producing T cells while maintaining protective mST2-expressing $\mathrm{T}$ cells during graft-versus-host disease. Sci Transl Med. 2015; 7: 308 ra160.

45. Hollmén M, Roudnicky F, Karaman S, Detmar M. Characterization of macrophage - cancer cell crosstalk in estrogen receptor positive and triple-negative breast cancer. Sci Rep. 2015; 5: 9188. 